Pacific Journal of Mathematics

THE HAAR INTEGRAL VIA NONSTANDARD ANALYSIS 


\title{
THE HAAR INTEGRAL VIA NON-STANDARD ANALYSIS
}

\author{
R. B. KIRK
}

\begin{abstract}
Since its discovery by A. Robinson in 1961, nonstandard analysis has been applied by an increasing number of authors to various parts of mathematics with the result that new and often more intuitively satisfying proofs for major theorems have been discovered. It is the purpose of this note to give a proof of the existence of a left invariant integral on a locally compact group using the methods of nonstandard analysis.
\end{abstract}

In a recent paper [2], $M$. Hausner indicated how some of the complications in one of the standard approaches to the existence of Haar measure can be streamlined using nonstandard analysis. The approach which will be presented here, on the other hand, develops the invariant integral from the beginning within the nonstandard framework.

As is well known, if $G$ is a discrete group, a left invariant integral on $G$ may be obtained by fixing a nonempty finite subset $A_{0} \subset G$ and than defining $\Phi(f)=1 / n\left(A_{0}\right) \sum_{x \in G} f(x)$ for every real-valued function $f$ with compact (i.e. finite) support on $G$. (Of course, $n\left(A_{0}\right)$ denotes the cardinal number of $A_{0}$.) If $G$ is not discrete, then in standard analysis the summation above becomes meaningless and other methods must be employed. However, it is not unreasonable to expect that in framework of nonstandard analysis it is possible to place a fixed amount of infinitesimal mass $1 / n\left(A_{0}\right)$ at each point of a set $A$ distributed uniformly throughout the group such that $\Phi(f)=\operatorname{st}\left(1 / n\left(A_{0}\right) \sum_{x \in A} * f(x)\right)$ is a left invariant integral. (Here $A_{0}$ should be a fixed *finite set.) This is basically the idea of the proof presented below. In the process of developing this idea, it is necessary to define precisely what is meant by a set being uniformly distributed. This is done by the notion of a maximal $d$-scattered subset of $G$ as defined below.

1. Topological groups. In this section several facts about topological groups will be collected for later reference. Let $N_{e}$ denote the neighborhood system at the identity $e$ of $G$. The left uniformity on $G$ is the uniform structure which has a base of entourages of the form $\left\{(x, y): y^{-1} x \in U\right\}$ for each $U \in N_{e}$. A pseudometric $d$ on $G$ is said to be compatible with the left uniformity if $\{(x, y): d(x, y)<\epsilon\}$ is an entourage of the left uniformity for every positive number $\epsilon$. The set 
of all pseudometrics on $G$ which are compatible with the left uniformity is denoted by $\Gamma$ and is called the gauge of the uniformity.

For $d_{1}, d_{2} \in \Gamma$ write $d_{1} \ll d_{2}$ if for every positive number $\epsilon$, there is a positive number $\delta$ such that for all $x, y \in G, d_{1}(x, y) \leqq \epsilon$ whenever $d_{2}(x, y) \leqq \delta$. An element $d \in \Gamma$ is left translation invariant if $d(z x, z y)=d(x, y)$ for all $x, y, z \in G$. The set of all left translation invariant elements of $\Gamma$ will be denoted by $\Gamma_{0}$. A proof of the following may be found in Hewitt and Ross [3] (Theorem 8.2, p. 68).

Proposition 1.1. Let $U \in N_{e}$. Then there is a $d \in \Gamma_{0}$ such that $\{x \in G: d(x, e)<1\} \subset U$.

As a corollary the following can be proved.

Proposition 1.2. 1. Let $d \in \Gamma$. Then there is a $d_{0} \in \Gamma_{0}$ with $d \ll d_{0}$.

2. If $d_{1}, d_{2} \in \Gamma_{0}$, then $d=\sup \left(d_{1}, d_{2}\right) \in \Gamma_{0}$ where $\sup \left(d_{1}, d_{2}\right)(x, y)=$ $\sup \left(d_{1}(x, y), d_{2}(x, y)\right)$ for all $x, y \in G$.

Proof. 1. Since $d_{1}, d_{2} \in \Gamma_{0}$, for every positive integer $n$, there is $U_{n} \in N_{e}$ such that $\left\{(x, y): y^{-1} x \in U_{n}\right\} \subset\{(x, y): d(x, y)<1 / n\}$. By Proposition 1.1, there is $d_{n} \in \Gamma_{0}$ such that $\left\{x: d_{n}(x, e)<1\right\} \subset U_{n}$. Since $d_{n}$ is left translation invariant, it follows that $\left\{(x, y): d_{n}(x, y)<1\right\} \subset$ $\left\{(x, y): y^{-1} x \in U_{n}\right\}$. Hence if $d_{n}(x, y)<1$, it follows that $d(x, y)<1 / n$.

For each $x, y \in G$, define,

$$
d_{n}^{\prime}(x, y)=\left\{\begin{array}{l}
d_{n}(x, y), \text { if } d_{n}(x, y) \leqq 1 \\
1, \text { otherwise }
\end{array}\right.
$$

It is easy to check that $d_{n}^{\prime} \in \Gamma_{0}$. Now for each $x, y \in G$, define $d_{0}(x, y)=$ $\sum_{n=1}^{\infty} 2^{-n} d_{n}^{\prime}(x, y)$. Then $d_{0} \in \Gamma_{0}$. Furthermore, if $d_{0}(x, y)<2^{-n}$, then $d_{n}(x, y)=d_{n}^{\prime}(x, y)<1$ so that $d(x, y)<1 / n$. Thus it follows that $d \ll d_{0}$.

2. Since $d_{1}, d_{2} \in \Gamma_{0}$, it is not difficult to verify that $d=\sup \left(d_{1}, d_{2}\right)$ is a left translation invariant pseudometric. For $\epsilon$ positive and $i=1,2$, there is $U_{i} \in N_{e}$ such that $\left\{(x, y): y^{-1} x \in U_{i}\right\} \subseteq\left\{(x, y): d_{i}(x, y)<\epsilon\right\}$. Then $U=U_{1} \cap U_{2} \in N_{e}$, and $\left\{(x, y): y^{-1} x \in U\right\} \subset\{(x, y): d(x, y)<\epsilon\}$. The proof is complete.

A real-valued function on $G$ is uniformly continuous if for every $\epsilon>0, \quad\{(x, y):|f(x)-f(y)|<\epsilon\} \quad$ is an entourage of the left uniformity. This is equivalent to $d_{f} \in \Gamma$ where $d_{f}$ is the pseudometric defined for all $x, y \in G$ by $d_{f}(x, y)=|f(x)-f(y)|$. The following is then an immediate corollary of Proposition 1.2. 
COROLlary 1.3. Let $f$ be a real-valued function on $G$. Then $f$ is uniformly continuous if and only if for every positive number $\alpha$ there is a $d \in \Gamma_{0}$ such that for all $x, y \in G,|f(x)-f(y)| \leqq \alpha$ whenever $d(x, y) \leqq 1$.

Proof. $(\Leftarrow)$ This is obvious.

( $\Rightarrow$ ) If $f$ is uniformly continuous, then $d_{f} \in \Gamma$. Hence by Proposition 1.2, there is a $d^{\prime} \in \Gamma_{0}$ with $d_{f} \ll d^{\prime}$. Hence if $\alpha$ is a positive number, there is a positive number $\delta$ such that for all $x, y \in G,|f(x)-f(y)| \leqq \alpha$ whenever $d^{\prime}(x, y) \leqq \delta$. Now define $d=\delta^{-1} d^{\prime}$ where $d(x, y)=$ $\delta^{-1} d^{\prime}(x, y)$ for all $x, y \in G$. Then clearly $d \in \Gamma_{0}$ and satisfies the requirements of the theorem. The proof is complete.

Let $d$ be a pseudometric on a set $S$. A subset of $A$ is said to be $d$-scattered if $d(x, y)>1$ for all $x, y \in A$ with $x \neq y$. The subset $A$ is maximally $d$-scattered if it is $d$-scattered and if $n(B) \leqq n(A)$ whenever $B$ is a $d$-scattered subset of $S$. (Of course, $n(Y)$ denotes the cardinal number of the set $Y$.) Recall that a subset $S$ of the group $G$ is bounded if for every $U \in N_{e}$, there is a finite set $T \subset S$ such that $S \subset$ $\cup\{x U: x \in T\}$. (In particular, every compact subset of $G$ is bounded.) It follows easily from Propositions 1.1 and 1.2 that $S$ is bounded if and only if for every $d \in \Gamma$ and for every positive number $\epsilon, S$ contains a finite $\epsilon$-net relative to $d$. (That is, there is a finite set $T \subset S$ with the property that for every $x \in S$, there is a $y \in T$ with $d(x, y) \leqq \epsilon$.

Proposition 1.4. Let $S$ be a non-empty bounded subset of $G$ and let $d \in \Gamma$. Then there is a positive integer $p$ such that $n(A) \leqq p$ for every $d$-scattered subset $A \subset S$. In particular, $S$ contains a finite maximally $d$-scattered subset.

Proof. Let $T$ be a finite $\epsilon$-net in $S$ relative to $d$ where $\epsilon=\frac{1}{2}$. Let $p=n(T)$. Assume that $A \subset S$ is $d$-scattered. Then $n(A) \leqq$ $p$. Indeed, assume that $p<n(A)$. For each $x \in A$, take $\varphi(x) \in T$ with $d(x, \varphi(x)) \leqq \epsilon$. Since $p<n(A)$, there are $x, y \in A$ with $x \neq y$ and $\varphi(x)=\varphi(y)$. Hence $d(x, y)>1$ since $A$ is $d$-scattered. However, since $\varphi(x)=\varphi(y), d(x, y) \leqq d(x, \varphi(x))+d(\varphi(y), y) \leqq 2 \epsilon=1$. This is a contradiction and the proof is complete.

2. The non-standard group $* G$. In this section properties of the nonstandard model ${ }^{*} G$ of $G$ which are needed to develop the Haar integral will be discussed. For background the reader is referred to the articles [4] and [5] by W. A. J. Luxemburg. Let ${ }^{*} G$ be an enlargement of the superstructure of $G \cup R$ (where $R$ is the set of real 
numbers) in the sense of A. Robinson [6]. This model will be fixed for the remainder of the paper. If $C_{u}$ denotes the set of real-valued, uniformly continuous functions, then the following holds.

Proposition 2.1. There is a positive infinitesimal $\alpha$ and there is a $d \in{ }^{*}\left(\Gamma_{0}\right)$ such that for all $x, y \in{ }^{*} G$, if $d(x, y) \leqq 1$, then $\left|{ }^{*} f(x)-{ }^{*} f(y)\right| \leqq \alpha$ for all $f \in C_{u}$.

Proof. Let $\mathscr{R} \subset\left(C_{u} \times R^{+}\right) \times\left(\Gamma_{0} \times R^{+}\right)$(where $R^{+}$denotes the set of positive real numbers) be the binary relation defined by $((f, \epsilon),(d, \alpha)) \epsilon$ $\mathscr{R}$ if and only if (i) $0<\alpha<\epsilon$ and (ii) for all $x, y \in G$, if $d(x, y) \leqq 1$, then $|f(x)-f(y)| \leqq \alpha$.

It is not hard to see that $\mathscr{R}$ is concurrent over its domain. Indeed, let $\left(f_{i}, \epsilon_{i}\right) \in C_{u} \times R^{+}$for $i=1, \cdots, n$. Let $\epsilon=\min \left(\epsilon_{1}, \cdots, \epsilon_{n}\right)$ and take $0<\alpha<\epsilon$. Also for each $i=1, \cdots, n$, choose, by Corollary 1.3, a $d_{i} \in \Gamma_{0}$ such that for all $x, y \in G$, if $d_{i}(x, y) \leqq 1$, then $\left|f_{i}(x)-f_{i}(y)\right| \leqq$ $\alpha$. Then by Proposition 1.2, $d=\sup \left(d_{1}, \cdots, d_{n}\right) \in \Gamma_{0}$. It follows that $\left(\left(f_{i} \epsilon_{i}\right),(d, \alpha)\right) \in \mathscr{R}$ for $i=1, \cdots, n$.

Since $\mathscr{R}$ is concurrent over its domain and since ${ }^{*} G$ is an enlargement, there is $(d, \alpha) \in *\left(\Gamma_{0}\right) \times^{*}\left(R^{+}\right)$such that $((* f, \epsilon),(d, \alpha)) \in * \mathscr{R}$ for every $f \in C_{u}$ and every $\epsilon \in R^{+}$. Thus $0<\alpha<\epsilon$ for all $\epsilon \in R^{+}$so that $\alpha$ is an infinitesimal. Furthermore, if $x, y \in{ }^{*} G$ and if $d(x, y) \leqq 1$, then $\left|{ }^{*} f(x)-{ }^{*} f(y)\right| \leqq \alpha$ for all $f \in C_{u}$. The proof is complete.

3. The marriage problem. The key to establishing translation invariance in the proof below is the solution to the so-called marriage problem. (See P. Halmos and H. Vaughn, [1].) Let two sets $A$ and $B$ (of boys and girls respectively) be given together with a relation $\mathfrak{A}$ (of acquaintance) between them. The marriage problem is said to have a solution if it is possible to marry every boy to a girl of his acquaintance. It is obvious that a necessary condition in order that the marriage problem have a solution is that every set of $k$ boys must have at least $k$ girls among their total acquaintance. The interesting fact is that this condition is also sufficient. The precise statement of the result is as follows. (For a proof the reader is referred to [1] or to [3], p. 248.)

Proposition 3.1. Let $A$ and $B$ be two nonempty sets with $A$ finite, and let $\mathfrak{A} \subset A \times B$. If $S \subset A$, then let $\mathfrak{U}(S)=$ $\{y \in B: \exists x \in S,(x, y) \in \mathfrak{A}\}$. There is a one-one function $\varphi$ from $A$ into $B$ such that $(x, \varphi(x)) \in \mathfrak{A}$ for all $x \in A$ if and only if $n(S) \leqq n(\mathfrak{U}(S))$ for every set $S \subset A$.

4. The Haar integral on a compact group. Since the proof of the existence of the Haar integral on a compact group contains 
all the essential ideas of the proof in the locally compact case and since it avoids some of the techincal complications, the proof of this special case will be given separately. Recall that a left invariant Haar integral on $G$ is a positive linear functional $\Phi$ defined on the space $C(G)$ (of continuous, real-valued functions) such that $\Phi\left({ }_{a} f\right)=\Phi(f)$ for all $a \in G$. (Of course, $f$ denotes the left translate of $f$ by $a$ defined by ${ }_{a} f(x)=f\left(a^{-1} x\right)$ for all $x \in G$.)

Let $d \in *\left(\Gamma_{0}\right)$ and let $\alpha$ be an infinitesimal such that for all $x, y \in{ }^{*} G$ if $d(x, y) \leqq 1$, then $\left|{ }^{*} f(x)-{ }^{*} f(y)\right| \leqq \alpha$ for all $f \in C(G)$. (Such a $d$ and $\alpha$ exist by Proposition 2.1 and the fact every $f \in C(G)$ is uniformly continuous.) By Proposition 1.4 and the fundamental theorem of nonstandard analysis, there is an internal, *finite $d$-scattered set $A \subset{ }^{*} G$ such that $n(B) \leqq n(A)$ for every internal $d$-scattered subset of $G$.

If $f \in C(G)$, then $f$ is bounded. This implies that the nonstandard number $1 / n(A) \sum_{x \in A} * f(x)$ is finite. It thus makes sense to define $\Phi(f)=\operatorname{st}\left(1 / n(A) \sum_{x \in A} * f(x)\right)$.

We can now prove the following.

Theorem 4.1. Let $A \subset^{*} G$ be as above and for each $f \in C(G)$, define $\Phi(f)=\operatorname{st}\left(1 / n(A) \Sigma_{x \in A} * f(x)\right)$. Then $\Phi$ is a left invariant integral on $G$.

Proof. It is obvious that $\Phi$ is a positive linear functional on $C(G)$. Hence all that needs to be shown is that $\Phi$ is left translation invariant. To this end fix $f \in C(G)$ and $a \in G$. Since $d \in *^{*}\left(\Gamma_{0}\right)$ is left translation invariant on ${ }^{*} G$, it follows that $a^{-1} A=\left\{a^{-1} x: x \in A\right\}$ is $d$-scattered in $G$. Define $\mathfrak{A} \subset a^{-1} A \times A$ by $(y, x) \in \mathfrak{A}$ if and only if $d(x, y) \leqq 1$. If $S$ is any internal subset of $a^{-1} A$ and if $\mathfrak{A}(S)=$ $\{x \in A: \exists y \in S,(y, x) \in \mathfrak{A}\}, \quad$ then $n(S) \leqq n(\mathfrak{H}(S))$. Otherwise, $n(\mathfrak{H}(S))<n(S)$ and $B=S \cup(A-\mathfrak{U}(S))$ is an internal $d$-scattered subset of $G$ with $n(A)<n(B)$. This is contrary to the fact that $A$ is maximally $d$-scattered. It now follows from the fundamental theorem of nonstandard analysis applied to Proposition 3.1 and from the fact that $n(A)=n\left(a^{-1} A\right)$, that there is an internal, one-one function $\varphi$ from $A$ onto $a^{-1} A$. Hence, it follows that:

$$
\begin{aligned}
\Phi\left(f-{ }_{a} f\right) & =\operatorname{st}\left(\frac{1}{n(A)} \sum_{x \in A}\left[{ }^{*} f(x)-*_{f}\left(a^{-1} x\right)\right]\right) \\
& =\operatorname{st}\left(\frac{1}{n(A)}\left[\sum_{x \in A} *_{f} f(x)-\sum_{y \in a^{-1} A} *^{*} f(y)\right]\right) \\
& \leqq \operatorname{st}\left(\frac{1}{n(A)} \sum_{x \in A}\left|{ }^{*} f(x)-*^{*} f(\varphi x)\right|\right) \\
& \leqq \operatorname{st}(\alpha)=0,
\end{aligned}
$$


since $d(x, \varphi x) \leqq 1$ implies that $|* f(x)-* f(\varphi x)| \leqq \alpha$. The proof is complete.

5. The Haar integral on a locally compact group. In this section $G$ is assumed to be locally compact, and $C_{0}(G)$ is used to denote the continuous functions of $G$ with compact support. Recall that $C_{0}(G) \subset C_{u}$. A left invariant Haar integral on $G$ is a positive linear functional $\Phi$ on $C_{o}(G)$ such that $\Phi\left({ }_{a} f\right)=\Phi(f)$ for all $f \in C_{0}(G)$ and all $a \in G$.

Definition. A function $\Psi: C_{0}(G)^{+} \rightarrow R$ is a left Haar functional if the following conditions hold:

(i) $0 \leqq \Psi(f)$ for all $f \in C_{0}(G)^{+}$.

(ii) $\Psi(\xi f)=\xi \Psi(f)$ for all $f \in C_{0}(G)^{+}$and all $\xi \in R^{+}$.

(iii) $\Psi(f+g)=\Psi(f)+\Psi(g)$, for all $f, g \in C_{0}(G)^{+}$.

(iv) $\Psi\left({ }_{a} f\right)=\Psi(f)$, for all $f \in C_{0}(G)^{+}$and all $a \in G$.

Proposition 5.1. Let $\Psi$ be a left Haar functional on $G$ and, for $f \in C_{0}(G)$, define $\Phi(f)=\Psi\left(f^{+}\right)-\Psi\left(f^{-}\right)$. Then $\Phi$ is a left invariant Haar integral on $G$.

Proof. The result is immediate from the identities, $(f+g)^{+}+f^{-}+$ $g^{-}=(f+g)^{-}+f^{+}+g^{+},\left({ }_{a} f\right)^{+}={ }_{a}\left(f^{+}\right)$and $\left({ }_{a} f\right)^{-}={ }_{a}\left(f^{-}\right)$.

It will now be shown how to define a left Haar functional within the context of the nonstandard group ${ }^{*} G$. By Proposition 2.1, take $d \in$ $*\left(\Gamma_{0}\right)$ and an infinitesimal $\alpha$ such that for all $x, y \in{ }^{*} G$, if $d(x, y) \leqq 1$, then $\left|{ }^{*} f(x)-{ }^{*} f(y)\right| \leqq \alpha$ for all $f \in C_{0}(G)$. Next fix a compact neighborhood $G_{0}$ of the identity in $G$. Then by Proposition 1.4, there is an internal, *finite maximally $d$-scattered subset $A_{0}$ of $*\left(G_{0}\right)$. For each $f \in C_{0}(G)$, let $S_{f}=*(\operatorname{supp} f)$ where supp $f$ denotes the support of $f$ in $G$. Since supp $f$ is compact, again by Proposition 1.4 there is an internal, *finite maximally $d$-scattered subset $A_{f}$ of $S_{f}$. Then the following holds.

Lemma 5.2. For all $f \in C_{0}(G)$, the nonstandard rational $n\left(A_{f}\right) / n\left(A_{0}\right)$ is finite.

Proof. Since supp $f$ is compact, there is a finite set $\left\{a_{1}, \cdots, a_{p}\right\} \subset$ supp $f$ with supp $f \subset \cup_{i=1}^{p} a_{i} G_{0}$. Hence, $S_{f} \subset \cup_{i=1}^{p} a_{i}{ }^{*}\left(G_{0}\right)$. Since $d$ is left translation invariant, $\left(a_{i}^{-1} A_{f}\right) \cap *\left(G_{0}\right)$ is a $d$-scattered subset of $*\left(G_{0}\right)$. Hence, it follows that $n\left(A_{f} \cap a_{i}{ }^{*}\left(G_{0}\right)\right)=n\left(a_{i}^{-1} A_{f} \cap *\left(G_{0}\right)\right) \leqq$ $n\left(A_{0}\right)$ for all $i=1, \cdots, p$. Thus,

$$
\begin{aligned}
n\left(A_{f}\right) & =n\left(\bigcup_{i=1}^{p}\left(A_{f} \cap a_{i}^{*}\left(G_{0}\right)\right)\right) \\
& \leqq \sum_{i=1}^{p} n\left(A_{f} \cap a_{i}^{*}\left(G_{0}\right)\right) \leqq p n\left(A_{0}\right) .
\end{aligned}
$$


Thus $n\left(A_{f}\right) / n\left(A_{0}\right) \leqq p$ where $p$ is a standard natural number. The proof is complete.

Now if $f \in C_{0}(G)$, then $|f|$ is bounded by some real number M. Hence

$$
\left|\frac{1}{n\left(A_{0}\right)} \sum_{x \in A_{f}} * f(x)\right| \leqq \frac{n\left(A_{f}\right)}{n\left(A_{0}\right)} M
$$

which is finite by the above lemma. It then makes sense to define for each

$$
f \in C_{0}(G)^{+}, \Psi(f)=\operatorname{st}\left(\frac{1}{n\left(A_{0}\right)} \sum_{x \in A_{f}} *_{f}(x)\right)
$$

TheOrem 5.3. The function $\Psi: C_{0}(G)^{+} \rightarrow R$ is a left Haar functional.

Proof. It is obvious that $0 \leqq \Psi(f)$ and that $\Psi(\xi f)=\xi \Psi(f)$ for all $f \in C_{0}(G)^{+}$and all $\xi \in R^{+}$. It remains to show that $\Psi\left({ }_{a} f\right)=\Psi(f)$ and that $\Psi(f+g)=\Psi(f)+\Psi(g)$ for all $f, g \in C_{0}(G)^{+}$and all $a \in G$. The remainder of the proof will be devoted to demonstrating these two properties.

(I) $\Psi\left({ }_{a} f\right)=\Psi(f)$ for all $f \in C_{0}(G)^{+}$and all $a \in G$.

Since $d$ is left translation invariant, $a^{-1} A_{a f}$ is an internal, $d$ scattered subset of $S_{f}$. Since $A_{f}$ is maximally $d$-scattered in $S_{f}, n\left(a^{-1} A_{a f}\right) \leqq$ $n\left(A_{f}\right)$. Similarly, since $A_{a^{s}}$ is maximally $d$-scattered in $S_{a^{\prime}}, n\left(A_{f}\right)=$ $n\left(a A_{f}\right) \leqq n\left(A_{a^{s}}\right)=n\left(a^{-1} A_{a^{s}}\right)$. Hence $n\left(A_{f}\right)=n\left(a^{-1} A_{a f}\right)$. By an application of Proposition 3.1 similar to that in the proof of Theorem 4.1, it follows that there is a one-one, internal function $\varphi$ from $A_{f}$ into $a^{-1} A_{\text {af }}$ such that $d(x, \varphi(x)) \leqq \alpha$ for all $x \in A_{f}$. It then follows that,

$$
\begin{aligned}
\left|\Psi(f)-\Psi\left({ }_{a} f\right)\right| & =\left|\operatorname{st}\left(\frac{1}{n\left(A_{0}\right)}\left[\sum_{x \in A_{f}} * f(x)-\sum_{x \in A_{a f}} * f\left(a^{-1} x\right)\right]\right)\right| \\
& =\operatorname{st}\left(\frac{1}{n\left(A_{0}\right)}\left|\sum_{x \in A_{f}} * f(x)-\sum_{y \in a^{-1} A_{a f}} * f(y)\right|\right) \\
& \leqq \mathrm{st}\left(\frac{1}{n\left(A_{0}\right)} \sum_{x \in A_{f}}|* f(x)-* f(\varphi(x))|\right) \\
& \leqq \mathrm{st}\left(\frac{n\left(A_{f}\right)}{n\left(A_{0}\right)} \alpha\right)=0
\end{aligned}
$$

by Lemma 5.2 and the fact that $\left|{ }^{*} f(x)-{ }^{*} f(y)\right| \leqq \alpha$ whenever $d(x, y)$ $\leqq 1$. Hence (I) follows. 
(II) $\Psi(f+g)=\Psi(f)+\Psi(g)$ for all $f, g \in C_{0}(G)^{+}$.

The proof of (II) is essentially combinatorial, but it is technically rather complicated. For this reason, it will be broken into a number of steps. Let $f \in C_{0}(G)^{+}$be fixed, and for each $0 \leqq \delta \in{ }^{*} R$, define $S^{\delta}=$ $\left\{x \in S_{f}: \delta \leqq * f(x)\right\}$.

(a) Let $g \in C_{0}(G)^{+}$and let $0 \leqq \delta \in * R$. Then there is an internal, one-one function $\varphi$ from $A_{f} \cap S^{\delta+2 \alpha}$ into $A_{f+g} \cap S^{\delta+\alpha}$ such that $d(x, \varphi x)$ $\leqq 1$ for all $x \in A_{f} \cap S^{\delta+2 \alpha}$.

Let $A=A_{f} \cap S^{\widetilde{\delta+2 \alpha}}, B=A_{f+g} \cap S^{\delta+\alpha}$ and

$$
\mathfrak{U}=\{(x, y): x \in A, y \in B \text { and } d(x, y) \leqq 1\}
$$

If $T$ is an internal subset of $A$, then $n(T) \leqq n(\mathfrak{H}(T))$ where $\mathfrak{U}(T)=$ $\{y \in B: \exists x \in T,(x, y) \in \mathfrak{A}\}$. Indeed, if this is not so, define $D=$ $T \cup\left(A_{f+g}-\mathfrak{A}(T)\right)$. Then $D$ is an internal subset of $S_{f+g}=S_{f} \cup S_{g}$ with $n\left(A_{f+g}\right)<n(D)$. It will now be shown that $D$ is $d$-scattered. Since $A_{f+g}$ is maximally $d$-scattered in $S_{f+g}$, it will then follow that $n(D) \leqq$ $n\left(A_{f+g}\right)$ which is a contradiction.

In order to show that $D$ is $d$-scattered, it is sufficient to show that if $x \in T$ and $y \in A_{f+g}-\mathfrak{A}(T)$, then $d(x, y)>1$. If $y \in S^{\delta+\alpha}$, then since $y \notin \mathfrak{A}(T), d(x, y)>1$ by the definition of $\mathfrak{A}$. Hence assume that $y \notin S^{\delta+\alpha}$ so that ${ }^{*} f(y)<\delta+\alpha$. Since $x \in T \subset S^{\delta+2 \alpha}, \delta+2 \alpha \leqq * f(x)$. Thus $* f(x)-*_{f}(y)>\alpha$. But if $d(x, y) \leqq 1$, then $|* f(x)-* f(y)| \leqq$ $\alpha$. Hence $d(x, y)>1$ as claimed.

Since $n(T) \leqq n(\mathfrak{A}(T))$ for every internal subset $T$ of $A$, it follows from Proposition 3.1 and the fundamental theorem of nonstandard analysis that there is an internal one-one function $\varphi$ from $A$ into $B$ such that $(x, \varphi x) \in \mathfrak{A}$ for all $x \in A$. The verification of (a) is complete.

(b) Let $g \in C_{0}(G)^{+}$and let $0 \leqq \delta \in *^{*}$. Then $n\left(A_{f} \cap S^{\delta+2 \alpha}\right) \leqq$ $n\left(A_{f+g} \cap S^{\delta+\alpha}\right) \leqq n\left(A_{f} \cap S^{\delta}\right)$.

The first inequality follows from (a). In order to see the second, assume that $n\left(A_{f} \cap S^{\delta}\right)<n\left(A_{f+g} \cap S^{\delta+\alpha}\right)$. Define $B=$ $\left(A_{f+g} \cap S^{\delta+\alpha}\right) \cup\left(A_{f}-S^{\delta}\right)$. If $x \in S^{\delta+\alpha}$ and $y \notin S^{\delta}$, then $d(x, y)>$ 1. (Indeed, ${ }^{*} f(x) \geqq \delta+\alpha$ and ${ }^{*} f(y)<\delta$ implies that $* f(x)-{ }^{*} f(y)>$ $\alpha$. But if $d(x, y) \leqq 1$, then $\left|* f(x)-{ }^{*} f(y)\right| \leqq \alpha$.) Hence $B$ is an internal, $d$-scattered subset of $S_{f}$ and so $n(B) \leqq n\left(A_{f}\right)$. This contradicts $n\left(A_{f}\right)<n(B)$ and (b) follows.

(c) There is an infinitesimal $0 \leqq \delta_{0} \in{ }^{*} R$ such that $\beta=$ $n\left(A_{f} \cap\left[S^{\delta_{0}}-S^{\delta_{0}+2 \alpha}\right]\right) / n\left(A_{f}\right)$ is infinitesimal.

First choose an infinitesimal $0<\epsilon \in{ }^{*} R$ such that $n \alpha<\epsilon$ for every standard positive integer $n$. (For instance, take $\epsilon=\sqrt{\alpha}$.) For $0 \leqq$ $\delta \in * R$, define $n(\delta)=n\left(A_{f} \cap\left[S^{\delta}-S^{\delta+2 \alpha}\right]\right)$. The set $P=$ $\{n(\delta): \delta \in[0, \epsilon]\}$ is an internal, nonempty subset of the nonstandard 
natural numbers and, as such, contains a minimal element $n\left(\delta_{0}\right)$ where $\delta_{0} \in[0, \epsilon]$. (Thus $\delta_{0}$ is infinitesimal.) One of the following holds:

(*) $\delta_{0}+n \alpha<\epsilon$, for every standard positive integer $n$ or

(**) $\delta_{0}-n \alpha>0$, for every standard positive integer $n$. Indeed, if neither $\left({ }^{*}\right)$ nor $\left({ }^{* *}\right)$ holds, then there is a standard positive integer $n_{0}$ with $\epsilon \leqq \delta_{0}+n_{0} \alpha$ and $0 \leqq n_{0} \alpha-\delta_{0}$. But then $\epsilon \leqq$ $\left(\delta_{0}+n_{0} \alpha\right)+\left(n_{0} \alpha-\delta_{0}\right)=2 n_{0} \alpha$ which contradicts the fact that $n \alpha<\epsilon$ for every standard positive integer $n$.

It will now be shown that (c) holds for $\delta_{0}$. Indeed let $p$ be a fixed standard positive integer. If $\left(^{*}\right)$ holds, then since $S^{\delta_{2}} \subset S^{\delta_{1}}$ if $\delta_{1} \leqq \delta_{2}$, it follows that

$$
\begin{aligned}
n\left(A_{f}\right) & =n\left(\bigcup_{r=0}^{p} A_{f} \cap\left[S^{\delta_{0}+2 r \alpha}-S^{\delta_{0}+2(r+1) \alpha}\right]\right) \\
& \geqq \sum_{r=0}^{p} n\left(A_{f} \cap\left[S^{\delta_{0}+2 r \alpha}-S^{\delta_{0}+2(r+1) \alpha}\right]\right) \\
& \geqq p n\left(A_{f} \cap\left[S^{\delta_{0}}-S^{\delta_{0}+2 \alpha}\right]\right),
\end{aligned}
$$

since $\delta_{0}+2 r \alpha<\epsilon$ for $r=1, \cdots, p$ by $(*)$ so that $n\left(\delta_{0}\right) \leqq n\left(\delta_{0}+2 r \alpha\right)$ for $r=1, \cdots, p$. On the other hand, if $\left({ }^{* *}\right)$ holds, then

$$
\begin{aligned}
n\left(A_{f}\right) & \geqq n\left(\bigcup_{r=0}^{p} A_{f} \cap\left[S^{\delta_{0}-2 r \alpha}-S^{\delta_{0}-2(r-1) \alpha}\right]\right) \\
& \geqq \sum_{r=0}^{p} n\left(A_{f} \cap\left[S^{\delta_{0}-2 r \alpha}-S^{\delta_{0}-2(r-1) \alpha}\right]\right) \\
& \geqq p n\left(A_{f} \cap\left[S^{\delta_{0}}-S^{\delta_{0}+2 \alpha}\right]\right),
\end{aligned}
$$

since $\delta_{0}-2 r \alpha>0$ for $r=1, \cdots, p$ by $\left(^{* *}\right)$ so that $n\left(\delta_{0}\right) \leqq n\left(\delta_{0}-2 r \alpha\right)$ for $r=1, \cdots, p$.

In either case, it follows that $n\left(A_{f} \cap\left[S^{\delta_{0}}-S^{\delta_{0}+2 \alpha}\right]\right) / n\left(A_{f}\right) \leqq 1 / p$ for every nonstandard positive integer $p$. Thus the verification of (c) is complete.

(d) Let $g \in C_{0}(G)^{+}$. Then there is an infinitesimal $\gamma$ such that $\left|\Sigma_{x \in A_{f}} * f(x)-\sum_{y \in A_{f+g}} * f(y)\right| \leqq n\left(A_{f}\right) \gamma$.

Let $\delta_{0}$ and $\beta$ be infinitesimals as in (c) above. By (a) there is an internal one-one function $\varphi$ from $A_{f} \cap S^{\delta_{0}+2 \alpha}$ into $A_{f+g} \cap S^{\delta_{0}+\alpha}$. For notational convenience, let $A=A_{f} \cap S^{\delta_{0}+2 \alpha}, \quad B=A_{f}-S^{\delta_{0}+2 \alpha}, \quad C=$ $\left(A_{f+g} \cap S^{\delta_{0}+\alpha}\right)-\varphi\left[A_{f} \cap S^{\delta_{0}+2 \alpha}\right]$ and $D=S_{f} \cap\left[A_{f+g}-S^{\delta_{0}+\alpha}\right]$. Note that $n(C) \leqq n\left(A_{f} \cap\left[S^{\delta_{0}}-S^{\delta_{0}+\alpha}\right]\right)$ as a consequence of (b). Also $n(D) \leqq$ $n\left(A_{f}\right)$ since $D$ is $d$-scattered in $S_{f}$. Since $A_{f}=A \cup B$ and since $S_{f} \cap A_{f+g}=\varphi\left[A_{f} \cap S^{\delta_{0}+2 \alpha}\right] \cup C \cup D$, the following holds. 


$$
\begin{aligned}
\left|\sum_{x \in A_{f}} * f(x)-\sum_{y \in A_{f+g}} * f(y)\right| \leqq \sum_{x \in A} \mid * f(x)-* f\left(\varphi(x)\left|+\sum_{x \in B}\right| * f(x) \mid\right. \\
\quad+\sum_{y \in C}|* f(y)|+\sum_{y \in D}|* f(y)| \\
\leqq n\left(A_{f}\right) \alpha+n\left(A_{f}\right)\left(\delta_{0}+2 \alpha\right)+n(C) M+n\left(A_{f}\right)\left(\delta_{0}+\alpha\right) \\
\leqq n\left(A_{f}\right)\left(2 \delta_{0}+4 \alpha\right)+M n\left(A_{f}-\left[S^{\delta_{0}}-S^{\delta_{0}+2 \alpha}\right]\right) \\
\leqq n\left(A_{f}\right)\left(2 \delta_{0}+4 \alpha+M \beta\right) \\
\leqq n\left(A_{f}\right) \gamma
\end{aligned}
$$

where $\gamma=2 \delta_{0}+4 \alpha+M \beta$ is infinitesimal. Hence (d) is verified.

It is now possible to prove (II). Since (d) holds for an arbitrary $f \in C_{0}(G)^{+}$, if $f, g \in C_{0}(G)^{+}$, then there are infinitesimals $\gamma_{1}$ and $\gamma_{2}$ such that,

$$
\left|\sum_{x \in A_{f}} * f(x)-\sum_{y \in A_{f+g}} * f(y)\right| \leqq n\left(A_{f}\right) \gamma_{1}
$$

and

$$
\left|\sum_{x \in A_{g}} * g(x)-\sum_{y \in A_{f+g}} * g(y)\right| \leqq n\left(A_{g}\right) \gamma_{2} .
$$

Thus it follows from (1) and (2) that,

$$
\begin{aligned}
|\Psi(f)+\Psi(g)-\Psi(f+g)|= & \mid \operatorname{st}\left(\frac { 1 } { n ( A _ { 0 } ) } \left[\sum_{x \in A_{f}} * f(x)+\sum_{x \in A_{g}} *^{*} g(x)\right.\right. \\
& \left.\left.-\sum_{y \in A_{f+g}}[* f(y)+* g(y)]\right]\right) \mid \\
\leqq & \operatorname{st}\left(\frac{1}{n\left(A_{0}\right)}\left|\sum_{x \in A_{f}} * f(x)-\sum_{y \in A_{f+8}} * f(y)\right|\right) \\
& +\operatorname{st}\left(\frac{1}{n\left(A_{0}\right)}\left|\sum_{x \in A_{g}} * g(x)-\sum_{y \in A_{f+g}} * g(y)\right|\right) \\
\leqq & \operatorname{st}\left(\frac{n\left(A_{f}\right)}{n\left(A_{0}\right)} \gamma_{1}\right)+\operatorname{st}\left(\frac{n\left(A_{g}\right)}{n\left(A_{0}\right)} \gamma_{2}\right) \\
\leqq & 0,
\end{aligned}
$$

since $n\left(A_{f}\right) / n\left(A_{0}\right)$ and $n\left(A_{g}\right) / n\left(A_{0}\right)$ are finite by Lemma 5.2. Hence $\Psi(f+g)=\Psi(f)+\Psi(g)$. The proof of Theorem 5.3 is complete. 


\section{REFERENCES}

1. Paul R. Halmos and Herbert E. Vaughan, The marriage problem, Amer. J. Math., 72 (1950), 214-215.

2. Melvin Hausner, On a non-standard construction of Haar measure, Comm. Pure Appl. Math., 25 (1972), No. 4, 403-405.

3. E. Hewitt and K. Ross, Abstract Harmonic Analysis, Volume I, Springer-Verlag, 1963.

4. Luxemburg, W. A. J., A general theory of monads, Applications of Model Theory ot Algebra, Analysis, and Probability (Holt, Rinehart and Winston, 1969), 18-86.

5. — What is non-standard analysis? Math. Montly, Part II, 8 (1973), 38-67.

6. A. Robinson, Non-standard analysis, (North Holland Publishing Co., 1966).

Received March 28, 1974.

SOUTHERN ILLINOIS UNIVERSITY 



\section{PACIFIC JOURNAL OF MATHEMATICS}

\section{EDITORS}

RICHARD ARENS (Managing Editor)

University of California

Los Angeles, California 90024

\section{J. DugundJI}

Department of Mathematics University of Southern California Los Angeles, California 90007

D. Gilbarg and J. Milgram

Stanford University

Stanford, California 94305

\section{ASSOCIATE EDITORS}
E. F. BECKENBACH
B. H. NeumanN
F. WoLF
K. YoshiDA

\section{SUPPORTING INSTITUTIONS}

\author{
UNIVERSITY OF BRITISH COLUMBIA \\ CALIFORNIA INSTITUTE OF TECHNOLOGY \\ UNIVERSITY OF CALIFORNIA \\ MONTANA STATE UNIVERSITY \\ UNIVERSITY OF NEVADA \\ NEW MEXICO STATE UNIVERSITY \\ OREGON STATE UNIVERSITY \\ UNIVERSITY OF OREGON \\ OSAKA UNIVERSITY
}

\author{
UNIVERSITY OF SOUTHERN CALIFORNIA \\ STANFORD UNIVERSITY \\ UNIVERSITY OF TOKYO \\ UNIVERSITY OF UTAH \\ WASHINGTON STATE UNIVERSITY \\ UNIVERSITY OF WASHINGTON \\ AMERICAN MATHEMATICAL SOCIETY
}

The Supporting Institutions listed above contribute to the cost of publication of this Journal, but they are not owners or publishers and have no responsibility for its contents or policies.

Mathematical papers intended for publication in the Pacific Journal of Mathematics should be in typed form or offset-reproduced (not dittoed), double spaced with large margins. Underline Greek letters in red, German in green, and script in blue. The first paragraph or two must be capable of being used separately as a synopsis of the entire paper. Items of the bibliography should not be cited there unless absolutely necessary, in which case they must be identified by author and Journal, rather than by item number. Manuscripts, in duplicate, may be sent to any one of the four editors. Please classify according to the scheme of Math. Reviews, Index to Vol. 39. All other communications should be addressed to the managing editor, or Elaine Barth, University of California, Los Angeles, California, 90024.

100 reprints are provided free for each article, only if page charges have been substantially paid. Additional copies may be obtained at cost in multiples of 50 .

The Pacific Journal of Mathematics is issued monthly as of January 1966. Regular subscription rate: $\$ 72.00$ a year (6 Vols., 12 issues). Special rate: $\$ 36.00$ a year to individual members of supporting institutions.

Subscriptions, orders for back numbers, and changes of address should be sent to Pacific Journal of Mathematics, 103 Highland Boulevard, Berkeley, California, 94708.

PUBLISHED BY PACIFIC JOURNAL OF MATHEMATICS, A NON-PROFIT CORPORATION Printed at Jerusalem Academic Press, POB 2390, Jerusalem, Israel.

$$
\begin{gathered}
\text { Copyright (C) } 1975 \text { Pacific Journal of Mathematics } \\
\text { All Rights Reserved }
\end{gathered}
$$




\section{Pacific Journal of Mathematics}

\section{Vol. 58, No. $2 \quad$ April, 1975}

Zvi Artstein and John Allen Burns, Integration of compact set-valued functions . . . . . . . . . 297

Mark Benard, Characters and Schur indices of the unitary reflection group $[321]^{3} \ldots \ldots \ldots . .309$

Simeon M. Berman, A new characterization of characteristic functions of absolutely continuous

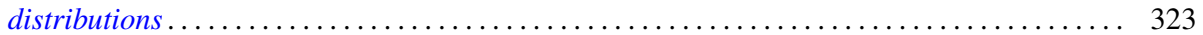

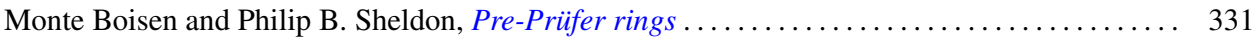

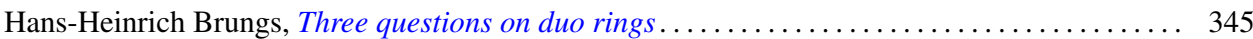

Iracema M. Bund, Birnbaum-Orlicz spaces of functions on groups................. 351

John D. Elwin and Donald R. Short, Branched immersions between 2-manifolds of higher

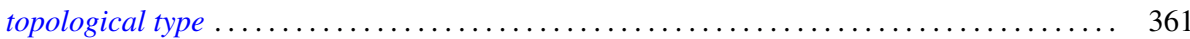

Eric Friedlander, Extension functions for rank 2, torsion free abelian groups . .......... 371

Jon Froemke and Robert Willis Quackenbush, The spectrum of an equational class of

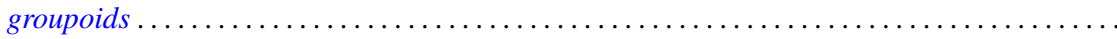

Barry J. Gardner, Radicals of supplementary semilattice sums of associative rings ...........

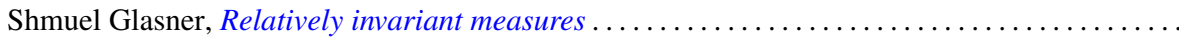

George Rudolph Gordh, Jr. and Sibe Mardesic, Characterizing local connectedness in inverse

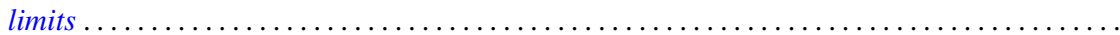

Siegfried Graf, On the existence of strong liftings in second countable topological spaces......

Stanley P. Gudder and D. Strawther, Orthogonally additive and orthogonally increasing

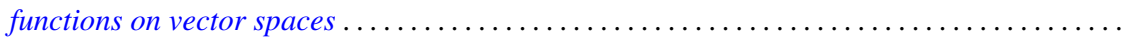

Darald Joe Hartfiel and Carlton James Maxson, A characterization of the maximal monoids and

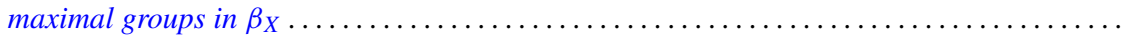

Robert E. Hartwig and S. Brent Morris, The universal flip matrix and the generalized faro-shuffle. .

William Emery Haver, Mappings between ANRs that are fine homotopy equivalences. .

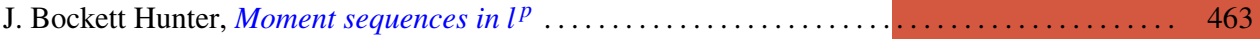

Barbara Jeffcott and William Thomas Spears, Semimodularity in the completion of a poset.... 467

Jerry Alan Johnson, A note on Banach spaces of Lipschitz functions . . . . . . . . . . . . 475

David W. Jonah and Bertram Manuel Schreiber, Transitive affine transformations on

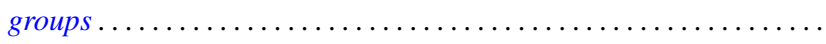

Karsten Juul, Some three-point subset properties connected with Menger's characterization of

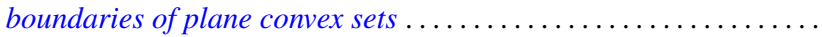

Ronald Brian Kirk, The Haar integral via non-standard analysis . . . . . . . . . . . . . 517

Justin Thomas Lloyd and William Smiley, On the group of permutations with countable

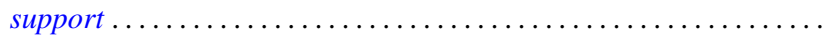

Erwin Lutwak, Dual mixed volumes .................................. 531

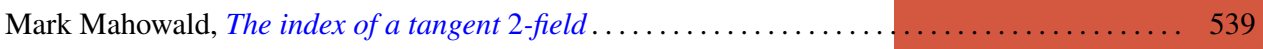

Keith Miller, Logarithmic convexity results for holomorphic semigroups . . . . . . . . . . . . 549

Paul Milnes, Extension of continuous functions on topological semigroups . . . . . . . . . . 553

Kenneth Clayton Pietz, Cauchy transforms and characteristic functions ................ 563

James Ted Rogers Jr., Whitney continua in the hyperspace $C(X) \ldots \ldots \ldots \ldots \ldots \ldots \ldots \ldots .569$

Jean-Marie G. Rolin, The inverse of a continuous additive functional . . . . . . . . . . . . 585

William Henry Ruckle, Absolutely divergent series and isomorphism of subspaces . ........ 605

Rolf Schneider, A measure of convexity for compact sets . ..................... 617

Alan Henry Schoenfeld, Continous measure-preserving maps onto Peano spaces .......... 627

V. Merriline Smith, Strongly superficial elements .......................... 643

Roger P. Ware, A note on quadratic forms over Pythagorean fields . . . . . . . . . . . . . . 651

Roger Allen Wiegand and Sylvia Wiegand, Finitely generated modules over Bezout rings . . . . 655

Martin Ziegler, A counterexample in the theory of definable automorphisms . . . . . . . . . 665 